\title{
Optimal Structural Topology of Materials with Micro-scale Tension-Compression Asymmetry Simulated Using Granular Micromechanics
}

\author{
Haipeng Jia ${ }^{\mathrm{a}}$, Anil Misra ${ }^{\mathrm{b}, \mathrm{c}^{*}}$, Payam Poorsolhjouy ${ }^{\mathrm{b}}$, Congyi Liu ${ }^{\mathrm{a}}$ \\ ${ }^{a}$ Department of Mechanical Engineering, Hebei University of Technology Tianjin, 300130, P. R. China, E- \\ mail: jiahaipeng@gmail.com \\ ${ }^{b}$ Civil, Environmental and Architectural Engineering Department, Bioengineering Research Center (BERC), \\ University of Kansas, 1530 W. 15th Street, Learned Hall, Lawrence, KS 66045-7609, USA \\ 'State Key Laboratory of Ocean Engineering, Department of Civil Engineering, Shanghai Jiao Tong \\ University, Dongchuan Road, Minhang, Shanghai200240, P. R. China \\ "Corresponding author. E-mail: amisra@ku.edu (A. Misra) \\ Tel.: +1 (785) 864-1750; fax: +1 (785) 864-1742.
}

Revised manuscript submitted for possible publication in:

Materials \& Design 


\begin{abstract}
Material systems derived from particulate precursor exhibit significant micro-scale tensioncompression asymmetry under loading which leads to macro-scale material anisotropy. Topology optimization of structures composed of such materials could suggest new unique topologies as well as material anisotropy distribution. This paper utilizes the granular micromechanics method that relates the micro-scale interactions to the macro-scale for defining material constitutive relationship. The resultant constitutive relationship is implemented in the evolutionary structure optimization algorithm based upon finite element analysis. Benchmark problems are simulated to investigate the influences of degrees of asymmetry on structure topologies and to illustrate how material anisotropy evolves under given boundary conditions.
\end{abstract}

\title{
Key Words
}

Tension-compression Asymmetry; Granular Micromechanics; Structure Topology Optimization; Evolutionary Structure Optimization; Finite Element Analysis 


\section{Introduction}

Materials formed from particulate precursors retain a granular nature such that their macro-scale behavior is significantly influenced by the grain-grain interactions. A distinctive characteristic of these materials is the effect of the asymmetric tension-compression grain-grain interaction upon their macro-scale anisotropy. This unique behavior trait therefore influences the optimal topology of structures formed from these materials as well as the material anisotropy distribution. With recent advances in numerical computation and optimization algorithms as well as in material and structural fabrication, optimal topologies with tailored material characteristics may be designed. Research in topology optimization has progressed rapidly since early 1980s (see for example [1-6]) and many new mathematical techniques and their variants have been introduced [7-9]. More recently, attempts have been made at combining structural topology optimization along with material design. For example, Zuo et al.[10] have proposed a topology optimization scheme for concurrent multi-scale design of composite macro-structure and material microstructure. Techniques such as rapid prototyping, 3D printing, or additive manufacturing can potentially provide the ability to fabricate structures composed of specific material microstructure for desired behavior at the "material-scale" as well as the "structural-scale". Along these lines, Leary et al.[11] have developed a novel method by applying topology optimization to additive manufacturing without requiring additional support material. Topology optimization has become increasingly popular in industrial application during the last decades due to the tremendous cost savings that can result when employed in the early stage of structure design.

Most past topology optimization applications have focused upon isotropic materials using the approaches of classical continuum mechanics without the consideration of the micro-structural effects. Optimized structures of isotropic material defined by single set of Lame's constants under symmetric loading and support conditions exhibit certain symmetries [12-16]. Such symmetries are absent when one considers materials whose tension-compression properties are not same. In more recent times, topology optimization of structures composed of materials with different macro-scale properties in tension and compression have been considered [17-30], although this aspect was first addressed by Prager in 1950s [31, 32]. Optimization of structures composed of materials in which tension-compression asymmetry is at the micro-scale, however, 
pose new challenges. Among these are the appropriate computational approaches, material constitutive relationships, especially those that incorporate the micro-scale effects, and the proper choice of the optimization algorithm. Figure 1 illustrates the possible computational approaches their length scale applicability and computational demand [33]. Continuum approach remains among the most feasible methods although many bottlenecks exist, when we consider structures in which micro-scale tension-compression asymmetry exists. The key problem is to find the relationship of stress and strain while performing updating operations within structural topology optimization.

For materials considered in this paper, the macro-scale behavior is influenced in a complex manner due to the tension-compression asymmetry that exists at micro- or grain-scale. Results show that the macro-scale material behavior depends upon the loading conditions experienced by each material point within a structure modeled as boundary value problem in continuum mechanics. From the micro-scale point of view, the material tension-compression asymmetry behavior depends upon the local strain state and manifests as anisotropy at the macro-scale. In this case, the constitutive equations derived using the granular micromechanics approach (see for example [34-36]) provide an effective bridge between the grain-interactions and macro-scale material behavior.

In the subsequent discussion, we first present a brief description of the structure topology optimization methodology used in this work, we then describe the novel adaptation of granular micromechanics based constitutive law accounting for the micro-scale tension-compression asymmetry, the implementation of the numerical algorithm is then described and finally a set of benchmark problems in topology optimization are presented and discussed with respect to the effects introduced by the degree of micro-scale tension-compression asymmetry. Results show that the newly derived material model results in optimal designs that are significantly different than those for the traditional material constitutive relationships due to the micro-scale the tension-compression asymmetry.

\section{Structure Topology Optimization using Granular Micromechanics}

2.1 Mathematical formulation of topology optimization 
There are two main branches for structural topology optimization, namely the gradient based and the non-gradient based methods. In the gradient based methods, mathematical models are derived to calculate the sensitivities of the design variables. However, for large-scale, nonlinear, and non-convex problems convergence is difficult to achieve in this method. In the non-gradient based approaches, the material is removed or included according to the prescribed criteria depending upon the problems [8]. Algorithms based upon these approaches have been applied widely to topology optimization that utilizes element-wise design variables. Among these algorithms, it is useful to take note of the homogenization method [5] and Solid Isotropic Material with Penalization (SIMP) method [37, 38]. In addition, the Evolutionary Structural Optimization (ESO) method [39-42] has been proposed to solve nonlinear topology optimization problems.

Let $\Omega$ be a bounded open set occupied by an elastic material as shown in figure 2 . The boundary $\partial \Omega$ is made of disjoint parts, $\partial \Omega=\Gamma_{D} \cup \Gamma_{N}$, where Dirichlet boundary conditions are imposed on $\Gamma_{D}$, and Neumann boundary conditions on $\Gamma_{N}$. All admissible topology are required to be a subset of a design domain $\Omega$. We denote by $f$ the vector-valued function of the volume forces and by $g$ that of the surface valued function of external loadings. The displacement field $u$ in $\Omega$ is thus the solution of following system of equations $g$ for the case that $\Omega$ is occupied by a linear isotropic elastic material with Hooke's law $A$,

$$
\left\{\begin{array}{ll}
-\operatorname{div}(A \varepsilon(u))=f, & \text { in } \Omega \\
u=u_{0}, & \text { on } \Gamma_{D} \\
(A \varepsilon(u))=g, & \text { on } \Gamma_{N}
\end{array} .\right.
$$

where the first equation is the equilibrium equation and second and third equations represent Dirichlet and Neumann boundary conditions respectively. The objective of the topology optimization of design domain, $\Omega$, is to find the function

$$
\chi(x)=\left\{\begin{array}{lll}
1 & \text { if } & x \in \Omega_{s} \\
0 & \text { if } & x \in \Omega / \Omega_{s}
\end{array} .\right.
$$

where $x$ denotes design variables.

We now consider the finite element formulation for the solution of Eq. (1), such that the displacement field $u$ satisfies the equilibrium equation written in its weak variation form. In this 
case, the topology optimization of structure to maximize stiffness $[3,40,41]$ can be specified as follows:

Maximize: $\quad J(u)=\frac{1}{2} u^{T} K u=\frac{1}{2} \sum_{e=1}^{N} u^{e T} k^{e} u^{e}$.

Subject to: $\quad a(u, v)=L(v)$.

$$
\begin{aligned}
& \left.u\right|_{\Gamma_{D}}=u_{0} \forall v \in U . \\
& V=\int d \Omega \leq V_{\max } .
\end{aligned}
$$

Here, $J(u)$ is the objective function, usually the strain energy of the structure. Where $u$ is the displacement vector, and $K$ is the global stiffness matrix. Further $u^{e}$ is the element displacement vector, $k^{e}$ element stiffness matrix, and $\mathrm{N}$ is the total number of elements within the whole structure. In addition, $V$ is the volume of the structure, which is constrained by a prescribed maximum amount of material volume $V_{\max }$. Further, the bilinear form $a(u, v)$ and the load linear form $L(v)$ are given as Eq. (7) and (8).

$$
\begin{aligned}
& a(u, v)=\int_{\Omega} C_{i j k l} \varepsilon_{i j}(u) \varepsilon_{k l}(v) d \Omega . \\
& L(v)=\int_{\Omega} p v d \Omega+\int_{\Gamma} \tau v d s . \\
& \varepsilon_{i j}(u)=\frac{1}{2}\left(\frac{\partial u_{i}}{\partial x_{j}}+\frac{\partial u_{j}}{\partial x_{i}}\right) .
\end{aligned}
$$

Throughout this paper, summation convention over repeated indices is implied, unless explicitly mentioned otherwise. For topology optimization of tension-compression granular material structure, the value of the bilinear form is calculated using granular micromechanics based stiffness tensor $C_{i j k l}$ which depends on the current strain tensor at each integration point of the numerical scheme for evaluating Eq. (7).

\subsection{Constitutive law based upon granular micromechanics}

As in figure 1, various computational techniques have been established that describe and predict the mechanics and physics of materials on many different length scales, from ab-initio and atomistic methods up to the molecular scale and Molecular Dynamics (MD), and further, all the way up to the largest length scale where continuum mechanics is used for modeling material 
behavior. Each method is appropriate for a particular, limited range of length scales. Using atomistic and molecular models, even with the computation and memory power of today's supercomputers, modeling remains limited to analysis of a few thousands of particles, making it inapplicable to large scale systems and structures $[43,44]$. On the other hand, as the material's behavior is highly dependent upon its microstructure and micromechanical properties, modeling the material without consideration of its unique micro-scale properties leads to neglect of important features. For solving this problem, the method of granular micromechanics presents a practical approach in which the material's behavior is derived by incorporating the behavior of all inter-granular mechanisms [36, 45-47]. In fact, the concern for the effect of micro-scale interactions upon the macro-scale behavior of a material point is traceable to the early developments of continuum mechanics in the works of Piola, Navier and Cauchy [48-50].

In the current work, the granular micromechanics model is adapted in a novel manner for obtaining constitutive law that relates the tension-compression asymmetry of the graininteractions to the macro-scale for the first time. As in figure 3, for cohesive tension-compression asymmetry material, the objective is to find the stiffness tensor. In this method, the material point is envisioned as a collection of grains, each interacting with its neighbors through different intergranular mechanisms. Inter-granular kinematic measures are calculated using different micromacro links based on the macroscopic kinematic measures (strain tensor in first gradient theory). Then local, inter-granular constitutive laws governing the behavior of grain-pair interactions are used to find the force components conjugate to the inter-granular kinematic measures. Finally equating the volume average of energy from all grain-pair interactions and the macroscopic deformation energy of the material point, the macroscopic stress tensor is calculated. The modeling approach is shown schematically in Figure 3.

In this approach, material macroscopic behavior is derived directly as a function of the interaction of grain-pairs within the material point. In the present paper, the focus is to model materials with micro-scale tension-compression asymmetry. A bilinear behavior with unequal microscopic stiffness coefficients in tension and compression is assumed as a first approximation of typically nonlinear asymmetric tension-compression micro-scale force-displacement relationship. The bilinear assumption is designed to mimic the more realistic nonlinear behavior without the need for computationally expensive iterations at every step. 
Relative displacement $\delta_{\mathrm{i}}$ between two nearest neighbor grains $\mathrm{n}$ and $\mathrm{p}$, ignoring the grain rotations, is written as

$$
\delta_{i}=u_{i}^{p}-u_{i}^{n}
$$

Where, $\mathrm{u}_{\mathrm{i}}$ is the particle displacement and superscripts refer to the interacting particles. To obtain the continuum description of this discrete model, we utilize the Taylor series expansion. Displacement of grain $\mathrm{p}$ is written as the Taylor series expansion of a neighbor grain, $\mathrm{n}$, as follows $[34,35]$ :

$$
u_{i}^{p}=u_{i}^{n}+u_{i, j}^{n}\left(x_{j}^{p}-x_{j}^{n}\right) .
$$

where terms of second and higher orders are neglected. It is also noted that the average displacement field, $\mathrm{u}$, is assumed to represent the whole displacement field of the material point and no fluctuation in the displacement is considered. However, considering both displacement fluctuations from average and also second gradient terms, result in a model for micromorphic media $[35,51,52]$ which can predict non-classical phenomena such as elastic wave dispersions and frequency band-gaps and include the Cosserat effects. These higher-order effects could be particularly germane to optimal design especially when the size of the sub-elements approach the characteristic dimension of the microstructure. Moreover, second gradient strain energies are relevant to the design of light fabrics $[53,54]$ and certain pantographic structures $[55,56]$. In the present paper, we are limiting the analysis to the classical first gradient theory for illustrating the effect of tension-compression asymmetry.

In Eq. 11, $u_{i, j}$ is the displacement gradient, and $x_{j}^{n}$ and $x_{j}^{p}$ are the position vectors of the centroids of grains $n$ and $p$, respectively. Thus, the relative displacement, $\delta_{i}$, between two nearest neighbor grains $n$ and $p$ representing the $\alpha$-th inter-granular interaction is given by

$$
\delta_{i}^{\alpha}=u_{i, j}^{n}\left(x_{j}^{p}-x_{j}^{n}\right)=u_{i, j}^{n} l_{j}^{\alpha}=\varepsilon_{i j} l_{j}^{\alpha}
$$

where $l_{j}^{\alpha}$ is an inter-granular branch vector joining the centroids of grain-pair $\mathrm{n}$ and $\mathrm{p}$. So it is seen that the relative displacement of two grains inside the material is derived as the projection of the macroscopic strain tensor in the direction of the inter-granular interaction vector and multiplied by the length of the branch vector. This relative displacement vector is further decomposed into the directions of a local coordinate system defined separately for each grain-pair interaction. The local coordinates system is composed of one normal axis, $n$, in the direction of the vector joining the two 
particle centroids and two orthogonal axes lying in the tangential plane whose normal vector is $\mathrm{n}$. Two interacting grains and the direction of their local coordinate axes with respect to the global coordinate system can be seen in figure 4.

The Cartesian components of these axes is the global coordinate system are

$$
\begin{aligned}
& n_{i}=\langle\cos \theta, \sin \theta \cos \phi, \sin \theta \sin \phi\rangle . \\
& s_{i}=\langle-\sin \theta, \cos \theta \cos \phi, \cos \theta \sin \phi\rangle . \\
& t_{i}=\langle 0,-\sin \phi, \cos \phi\rangle .
\end{aligned}
$$

Using the defined coordinate system, the displacement vector, $\delta_{\mathrm{i}}$, is decomposed into components along the $\mathbf{n}, \mathbf{s}$, and $\mathbf{t}$ coordinate axes as follows

$$
\delta_{n}^{\alpha}=\delta_{i}^{\alpha} n_{i}^{\alpha} ; \quad \delta_{s}^{\alpha}=\delta_{i}^{\alpha} s_{i}^{\alpha} ; \quad \delta_{t}^{\alpha}=\delta_{i}^{\alpha} t_{i}^{\alpha}
$$

where $\delta_{n}^{\alpha}, \delta_{s}^{\alpha}$, and $\delta_{t}^{\alpha}$ are scalar values and represent one normal and two tangential components of inter-granular displacement. It is also noted that the superscripts $\mathrm{n}, \mathrm{s}$, and $\mathrm{t}$ in Eq. 14 do not follow index summation conventions. Conjugate to these displacement components, inter-particle force components are defined which are obtainable using a simple force law in the inter-particle scale. Normal and tangential stiffness coefficients are used to define the normal and tangential force components.

$$
\left\{\begin{array}{l}
f_{n}^{\alpha} \\
f_{s}^{\alpha} \\
f_{t}^{\alpha}
\end{array}\right\}=\left[\begin{array}{ccc}
k_{n}^{\alpha} & 0 & 0 \\
0 & k_{s}^{\alpha} & 0 \\
0 & 0 & k_{t}^{\alpha}
\end{array}\right]\left\{\begin{array}{l}
\delta_{n}^{\alpha} \\
\delta_{s}^{\alpha} \\
\delta_{t}^{\alpha}
\end{array}\right\} .
$$

Note that more complicated, non-linear, damage, plasticity, and damage-healing models of the material behavior are also attainable by incorporating non-linear force laws $[34,36,57,58]$. However this study focuses on the bilinear analysis of force-displacement relationships. Thus in order to model the effect of tension-compression asymmetry in macro-scale in a systematic fashion, the inter-particle stiffness is specified as follows:

$$
\begin{array}{ll}
k_{n}^{T \alpha}=\lambda k_{n}^{\alpha} ; k_{s}^{T \alpha}=\lambda k_{s}^{\alpha} ; & \text { for } \delta_{n}^{\alpha}>0 \\
k_{n}^{C \alpha}=k_{n}^{\alpha} ; \quad k_{s}^{C \alpha}=k_{s}^{\alpha} ; & \text { for } \delta_{n}^{\alpha} \leq 0 .
\end{array}
$$

where the superscript $\mathrm{T}$ and $\mathrm{C}$ denote Tension and Compression, respectively, and $\lambda$ is a constant between 0 and 1 . If $\lambda$ is equal to 1 , the material's behavior in tension is identical to that in compression and if it is equal to zero, the material has no tensile stiffness or strength. It is seen 
that both normal and tangential stiffness coefficients are functions of the normal component of displacement. The normal component of displacement is, as it is seen from Eq. 12 and 14, is a function of the direction of the grain-pair interaction. So, it is clear that from the very beginning of the loading process, grain-pair interactions in different directions will have different stiffness coefficients thus creating an induced anisotropy in the material [34, 36].

In order to write the force components in the global coordinate system, rotation tensor is defined as follows for each inter-granular contact

$$
T^{\alpha}=\left[\begin{array}{lll}
n_{1} & s_{1} & t_{1} \\
n_{2} & s_{2} & t_{2} \\
n_{3} & s_{3} & t_{3}
\end{array}\right] .
$$

Thus, the inter-granular force-displacement relationship can be written as

$$
f_{i}^{\alpha}=K_{i j}^{\alpha}\left(\delta_{p}^{\alpha}\right) \delta_{j}^{\alpha} ; \quad K_{i j}^{\alpha}=T_{i p}^{\alpha} k_{p q}^{\alpha} T_{j q}^{\alpha}
$$

where $K_{i j}\left(\delta_{p}\right)$ denotes that the inter-granular stiffness tensor as a function of relative displacement $\delta_{p}$ in the global coordinate system 123, while $k_{p q}$ represents the inter-granular stiffness tensor in grain's local axis nst and is shown in Eq. 15. It should be noted that the local stiffness tensors, $k_{i j}$ and $K_{p q}$, depend upon inter-granular displacement as given by Eq. (16). Using the principle of virtual work and equating the internal work done by all of the grain-pairs and that done by the stress and strain tensors and using Eq. 12, one will get

$$
\sigma_{i j} \varepsilon_{i j}=\frac{1}{V} \sum_{\alpha}\left(f_{i}^{\alpha} \delta_{i}^{\alpha}\right)=\frac{1}{V} \sum_{\alpha}\left(f_{i}^{\alpha}\left(\varepsilon_{i j} l_{j}^{\alpha}\right)\right)=\left(\frac{1}{V} \sum_{\alpha}\left(f_{i}^{\alpha} l_{j}^{\alpha}\right)\right) \varepsilon_{i j} \Rightarrow \sigma_{i j}=\frac{1}{V} \sum_{\alpha}\left(f_{i}^{\alpha} l_{j}^{\alpha}\right) .
$$

Now substituting Eq. 18 and then Eq. 12 into Eq. 19 one would derive the stress tensor in terms of strain tensor and the stiffness tensor.

$$
\sigma_{i j}=\frac{1}{V} \sum_{\alpha} K_{i k}^{\alpha}\left(\delta_{p}^{\alpha}\right) \delta_{k}^{\alpha} l_{j}^{\alpha}=\frac{1}{V} \sum_{\alpha} K_{i k}^{\alpha}\left(\delta_{p}^{\alpha}\right) \varepsilon_{k l} l_{l}^{\alpha} l_{j}^{\alpha}=\left(\frac{1}{V} \sum_{\alpha} K_{i k}^{\alpha}\left(\delta_{p}^{\alpha}\right) l_{l}^{\alpha} l_{j}^{\alpha}\right) \varepsilon_{k l}=C_{i j k l} \varepsilon_{k l} .
$$

Thus it is clearly seen that the stiffness tensor is derived using the local stiffness parameters as

$$
C_{i j k l}=\frac{1}{V} \sum_{\alpha} K_{i k}^{\alpha}\left(\delta_{p}^{\alpha}\right) l_{l}^{\alpha} l_{j}^{\alpha}
$$

Where the summation is done over all inter-granular contacts. It is also noted that since the intergranular stiffness tensor $K_{i k}\left(\delta_{p}\right)$ is a function of relative displacement $\delta_{p}$, it follows from Eq. 12 that the stiffness tensor is a function of strain. Using a probability density distribution function, 
$\xi(\theta, \phi)$, which defines the distribution of grain-pair contact properties in different direction [57], the summation is changed into integration over all generic directions as

$$
C_{i j k l}=l^{2} N_{p} \int_{\theta=0}^{\pi} \int_{\phi=0}^{2 \pi} K_{j k}\left(\delta_{p}^{\alpha}\right) n_{i} n_{l} \xi \sin \theta d \phi d \theta .
$$

where $N_{p}$ is the number of contacts in a unit volume of the material and $l$ is the nominal grain size (For a more detailed description of the transformation of the summation in Eq. 21 to the integration in Eq. 22, see paper [58, 59]). The probability density distribution function used for modeling materials which are inherently isotropic is a constant number for all directions and is defined as $\xi(\theta, \phi)=\frac{1}{4 \pi}$. However, for modeling materials with inherent anisotropy, it is possible to use other forms of the probability density distribution function that represents the distribution of stiffness and grain shapes in different directions in a material. In any case, the function used for the probability distribution function should be normalized such that $\int_{\theta=0}^{\pi} \int_{\phi=0}^{2 \pi} \xi \sin \theta d \phi d \theta=1$.

Misra and Poorsolhjouy [59] have shown that by using spherical harmonic expansions as the distribution function, it is possible to model materials with all different levels of inherent anisotropy. As mentioned, the asymmetric tension-compression micro-scale stiffness will result in anisotropic constitutive tensor depending upon the strain condition of the material point. Recently a more general quantitative measure of the degree of anisotropy termed as the universal anisotropy index has been introduced [59,60], and it is defined as

$$
A^{U}=5 \frac{G^{V}}{G^{R}}+\frac{K^{V}}{K^{R}}-6 \geq 0
$$

In Eq. 23 the superscripts $\mathrm{V}$ and $\mathrm{R}$ represent the Voigt and Reuss estimates of the shear, G, and, bulk, $\mathrm{K}$, moduli respectively, calculated using the updated constitutive tensor for each material point. The anisotropy index takes the value of 0 for isotropic materials and positive values for anisotropic materials.

\subsection{Computational Flow and its Numerical Implementations}

The topology optimization proceeds by modifying the layout of the material in design domain to obtain the optimal objective function. In this work, the objective function is taken as the strain energy of structure and the structure topology optimization is to find its minimum in order to 
attain the stiffest structure possible with boundary conditions and specified amount percent of material. Evolutionary Structural Optimization (ESO) [41, 42] was used to solve the mathematical topology optimization formulation. The Evolutionary Structure Optimization (ESO) is advantageous for solving many engineering design analysis and optimization problems where design sensitivity information is computationally intensive, or not available readily during design optimizations. For the cohesive granular material structure topology optimization proposed in this paper, the derivative of strain energy with respect to design variable cannot be obtained analytically. Therefore, the non-gradient based topology ESO algorithm is a preferred approach. This kind of bio-inspired algorithm has been applied to the shape optimization and topology optimization of physical problems [61, 62], such as compliant mechanisms, heat transfer, and energy harvesting systems.

For topology optimization, ESO method is based on the concept of gradually removing unnecessary or inefficient material from the structure, or gradually adding material at high strain energy area, to achieve an optimal design or topology. The value of optimization criterion function is calculated for each element and element removal is applied to those with low criteria values. The criterion used is the strain energy of each element termed as the sensitivity[63].

$\beta^{i}=\int_{\Omega^{e}} C_{i j k l} \varepsilon_{i j}(u) \varepsilon_{k l}(v) d \Omega$.

where for the given material point, the stiffness tensor $C_{i j k l}$ can be computed through Eq. (22) and as a function of inter-granular stiffness coefficients and the local strain. The low strain energy elements are removed iteratively according to rejection ratio, which is defined as the ratio of the number of elements removed at each iteration to the total number of elements in the initial finite element model, specified as $2 \%$ in this work. The procedure proceeds until the strain energy is minimized with constrained given material satisfied.

The main computational steps in the optimization algorithm are elucidated as follows:

Step1. Initialization of the parameters: Specify the input geometry parameters, boundary conditions and optimization initial parameters, such as volume constraint, penalty factor, tension -compression asymmetry ratio;

Step2. Solution of the equilibrium equation of structure: FEA (Finite Element Analysis)[64, 65] is adopted to compute the displacement field and strain energy; 
Step3. Computation of the sensitivity of candidate elements: The strain energy within an element is computed using Eq. 24;

Step4. Material addition and deletion: Remove the elements whose strain energy belongs to the bottom $2 \%$ from throughout the structure. Add elements proximal to the elements lying on the boundary of the topology whose strain energy is in top $2 \%$ based upon the criterion discussed in [40, 42];

Step5. Update material constitutive tensor of each element: Based upon the element strain, the element stiffness matrix is computed using the theory developed in section 2.3.

Step6. Output evolution of the structure topology and field variables, such as, nodal displacements, averaged Von Mises and principal stresses, and anisotropy indices.

Step7. Convergence check: If volume constraint is met, the iteration is completed; otherwise repeat Step 2 to Step 6 until convergence.

\section{Numerical Examples}

Four benchmark examples demonstrating the effectiveness of the proposed mathematical formulation of topology optimization for the cohesive granular material structure are presented. For each benchmark example, the topologies at key steps and their optimization history curve are given to demonstrate the topology variation. Additionally, the effect of $\lambda$ on the topology is studied to show the effect of different micro-scale tension-compression asymmetry on optimal topology. The objective function curve is also given for reference. In these calculations, the

material is assumed to be isotropic in the unloaded condition such that $\xi(\theta, \phi)=\frac{1}{4 \pi}$, with an unloaded Young's modulus of $52 \mathrm{GPa}$ and Poisson's ratio of 0.11 such that the stiffness parameters $k_{n}=2 \mathrm{kN} / \mathrm{m}$ and $k_{s}=1 \mathrm{kN} / \mathrm{m}$, the grain size $l=10 \mu \mathrm{m}$, and $\mathrm{N}_{\mathrm{p}}=10^{18} \mathrm{~m}^{-3}$.

\subsection{Topology optimization of cantilever in plane stress}

The cantilever beam problem is one of the benchmark structure topology optimization problems. The design objective is to minimize strain energy while satisfying volume constraints. The design domain and boundary condition is illustrated in figure 5. The left edge is fixed and a concentrated force $\mathrm{P}=1 \mathrm{~N}$ is applied at the middle point of right edge in the downward direction. 
In this example, the volume constraint is set at $60 \%$ of the total design domain. The design domain is discretized with $128 \times 80$ four-node plane stress elements.

The topologies of key steps during optimization are shown in figure 6 for $\lambda=0.4$. The color scheme corresponds to anisotropy index given in Eq. 23 which serves as a single parametric measure of material anisotropy (see reference $[59,60]$ ). As the optimization proceeds, it can be seen that both the material anisotropy distribution and the topology adjusts at each step. The anisotropy develops in response to the strain distribution in the evolved structure. A new constitutive tensor is computed at every material point based upon its strain since the intergranular stiffness tensor $K_{i k}\left(\delta_{p}\right)$ is a function of relative displacement $\delta_{p}$, and hence, a function of strain, as indicated in Eq. 22.. In general, under loading, some grain-pairs are under tension while others under compression depending upon the given strain condition as given by Eq. (12). Thus, the grain-pair stiffness will be different for different directions leading to a unique anisotropy at every material point. Locations with greater deviatoric strain will likely develop higher anisotropy and, in the subsequent step, the topology will evolve according the new material property distribution. The topology evolution process is clearly nonlinear although at each iteration step a linear boundary value problem is solved.

The displacement field of the optimal structure satisfying the KKT condition is given in figure 7a. Despite the well-developed mathematical background of a compliance minimization topology optimization problem, the problem statements are usually not representative of practical structural design requirements. One of the most important failure criteria is the von Mises stress, which can be constrained not to exceed the yield stress of the material. The averaged equivalent stress of the optimal structure is shown in figure 7b. Furthermore, the first principle stress, shown in figure $7 \mathrm{c}$, indicates the regions under tension and compression. It can be seen that the compressive regions which have higher stiffness (as shown in Eq. 16) have a larger size, compared to the tensile regions. In fact, the optimum structure consists more of compressive (strong) components and significantly less of tensile (weak) components. Such a result is expected, since stiffer regions can support larger proportion of the applied loading. Finally in 
figure 8 , the iteration history is depicted to illustrate the convergence of the solution in terms of strain energy as well as the volume constraint.

Moreover, to demonstrate the effect of varying the tension to compression stiffness ratio, $\lambda$, figure 9 shows the optimal topologies at different values of $\lambda$. The asymmetry of the overall structure as ratio, $\lambda$, decreases from 1 to 0.1 for same volume constraint, is remarkable. For ratio, $\lambda=1$, the classical results are found in which the structure has a symmetry about the horizontal axis. As the micro-scale tensile stiffness diminishes, the material softens at the macro-scale. For optimal distribution of strain energy, the structure responds by removing the softer elements and retaining (or adding) stiffer elements; a result which is not necessarily intuitive. In addition, different topologies achieved by varying the volume constraint for $\lambda=0.4$ are illustrated in figure 10. It is noteworthy that as the volume constraint is reduced from $90 \%$ to $20 \%$, the compliance nonlinearly increases from 1.25 to 4.25 . In all these cases though, the optimized structure is asymmetric about the horizontal axis and contains elements that appear to support the tensile arch.

\subsection{Topology optimization of cantilever under corner loading}

For this example, sown in figure 11, the left edge is fixed and force is applied at the lower corner of right edge in the downward direction. The volume constraint is set at $60 \%$ of the total design domain. The design domain is discretized with $128 \times 80$ four node plane stress elements. The key-steps during optimization are shown in figures 12 for $\lambda=0.3$. In figure 13 , the iteration history is depicted to illustrate the convergence of the solution in terms of strain energy as well as the volume constraint and the effect of the tension to compression stiffness ratio, $\lambda$ on final topology. In this case also as the ratio, $\lambda$, decreases from 1 to 0.1 for same volume constraint, the softer (tensile) elements are removed and stiffer (compressive) elements are added.

\subsection{Topology optimization of Michell structure}

Figure 14 describes one of the widely known benchmark problems in topology optimization. The Michell structure with a vertical forced applied on the middle point of the lower side. The parameters of geometry and boundary conditions have been illustrated in the figure. The design domain is discretized with $160 \times 80$ four node plane stress elements. The key-steps during optimization are shown in figures 15 . The effect of the tension to compression stiffness ratio, $\lambda$, 
is given in figure 16. The first principle stress field is given in figure 16 and as previously indicates that regions under compression are larger than those under tension.

\subsection{Topology optimization of MBB beam}

Widely used as benchmark problem for topology optimization, MBB beam is named from the Munich company Messerschmitt-Bölkow-Blom where the example was initially proposed. Figure 18 shows an MBB beam design problem and the ratio of length to height is 6 . To save CPU time half of the structure is optimized and the design domain is discretized with $180 \times 60$ four node plane stress elements. The key-steps during optimization are shown in figures 18 , and the first principle stress is given in figure 18, the region in blue indicates structure under compression are larger than those under tension for $\lambda=0.2$. The effect of the tension to compression stiffness ratio, $\lambda$, is shown in figure 19. Results in figure 19 show that the Von Mises stress field of the optimal structure satisfying the KKT condition.

\section{Concluding remarks}

Material systems that exhibit significant tension-compression asymmetry at the micro-scale develop anisotropic macro-scale behavior under loading. Since the strain condition is different at every point of a structure subjected to particular boundary conditions, each material point develops a different anisotropic behavior. The topology optimization of structures composed of such materials systems therefore lead to unique solutions that depend upon the degree of tensioncompression asymmetry. In this paper, a novel adaptation of the granular micromechanics method has been developed for obtaining the constitutive relationship for each material point. In this method it is possible to model the material's macroscopic behavior directly from the microscopic behavior of grain-pair interactions for a given strain condition. The constitutive model is implemented within the evolutionary structure optimization technique in which the objective function is to minimize the mean compliance of the structure. A set of numerical benchmark problems in structure topology optimization are computed and the asymmetry of final topology illustrates the validity and effectiveness of the proposed algorithm. 
The optimal topology is found, optimization history of objective compliance and constraint are given, and the effect of ratio of tension to compression stiffness coefficients on the optimum topology is analyzed. The topology configurations are clear, rational, and practical. The resultant topologies for all the benchmark problems show that the proposed mathematical formulation for structure topology optimization is computationally feasible.

The four benchmark examples demonstrate clearly the effects of tension-compression asymmetry which is expected for a vast variety of engineering as well as natural materials. The optimized topologies are vastly different than that obtained from classical simulations in which material isotropy is enforced and no account is made of the induced anisotropy that emerges as the material is subjected to loading. Similar effects may be observed in biological and other natural structures. Bones and other hard tissues are known to respond to loading [66-69] and the proposed approach can be used for understanding biomaterial structure and function, particularly in relation to their anisotropy [70]. Finally, it is noteworthy that the granular micromechanics can lead to general micromorphic continuum models [35] that exhibit the length scale effects of material microstructure and can lead to further modifications in the optimal design [71].

\section{Acknowledgments}

This work was supported partially by National Science Foundation (CMMI-1068528), National Science Foundation of China (51475137) and key project from Hebei Education Bureau (QN20131097). Their financial contribution is gratefully acknowledged.

\section{References}

[1] G.I.N. Rozvany, A critical review of established methods of structural topology optimization, Structural and Multidisciplinary Optimization 37(3) (2009) 217-237.

[2] J.D. Deaton, R.V. Grandhi, A survey of structural and multidisciplinary continuum topology optimization: post 2000, Structural and Multidisciplinary Optimization 49(1) (2014) 1-38. 
[3] H.A. Eschenauer, N. Olhoff, Topology optimization of continuum structures: A review*, Applied Mechanics Reviews 54(4) (2001) 331-390.

[4] G.I. Rozvany, T. Lewiński, Topology optimization in structural and continuum mechanics, Springer2014.

[5] M.P. Bendsøe, N. Kikuchi, Generating optimal topologies in structural design using a homogenization method, Computer methods in applied mechanics and engineering 71(2) (1988) 197-224.

[6] B. Hassani, E. Hinton, A review of homogenization and topology optimization III-topology optimization using optimality criteria, Computers \& structures 69(6) (1998) 739-756.

[7] H. Jia, H. Beom, Y. Wang, S. Lin, B. Liu, Evolutionary level set method for structural topology optimization, Computers \& Structures 89(5) (2011) 445-454.

[8] N.M. Patel, D. Tillotson, J.E. Renaud, A. Tovar, K. Izui, Comparative study of topology optimization techniques, AIAA journal 46(8) (2008) 1963-1975.

[9] N.P. van Dijk, K. Maute, M. Langelaar, F. Van Keulen, Level-set methods for structural topology optimization: a review, Structural and Multidisciplinary Optimization 48(3) (2013) 437-472.

[10] Z.H. Zuo, X. Huang, J.H. Rong, Y.M. Xie, Multi-scale design of composite materials and structures for maximum natural frequencies, Materials \& Design 51 (2013) 1023-1034.

[11] M. Leary, L. Merli, F. Torti, M. Mazur, M. Brandt, Optimal topology for additive manufacture: a method for enabling additive manufacture of support-free optimal structures, Materials \& Design 63 (2014) 678-690.

[12] X. Guo, Z. Du, G. Cheng, C. Ni, Symmetry properties in structural optimization: some extensions, Structural and Multidisciplinary Optimization 47(6) (2013) 783-794.

[13] X. Guo, C. Ni, G. Cheng, Z. Du, Some symmetry results for optimal solutions in structural optimization, Structural and Multidisciplinary Optimization 46(5) (2012) 631-645.

[14] G.I. Rozvany, On symmetry and non-uniqueness in exact topology optimization, Structural and Multidisciplinary Optimization 43(3) (2011) 297-317.

[15] K. Zhou, Topology optimization of truss-like continuum structures for natural frequencies, Structural and Multidisciplinary Optimization 47(4) (2013) 613-619.

[16] K. Zhou, X. Li, Topology optimization of truss-like continua with three families of members model under stress constraints, Structural and Multidisciplinary Optimization 43(4) (2011) 487-493.

[17] W. Achtziger, Truss topology optimization including bar properties different for tension and compression, Structural optimization 12(1) (1996) 63-74.

[18] P. Duysinx, Topology optimization with different stress limit in tension and compression, Third World Congress of Structural and Multidisciplinary Optimization (WCSMO3), 1999.

[19] M. Bruggi, P. Duysinx, A stress-based approach to the optimal design of structures with unilateral behavior of material or supports, Structural and Multidisciplinary Optimization 48(2) (2013) 311-326.

[20] M. Bruggi, Finite element analysis of no-tension structures as a topology optimization problem, Structural and Multidisciplinary Optimization 50(6) (2014) 957-973.

[21] M. Bruggi, A. Taliercio, Analysis of no-tension structures under monotonic loading through an energy-based method, Computers \& Structures 159 (2015) 14-25.

[22] P. Dewhurst, A general optimality criterion for strength and stiffness of dual-material-property structures, International journal of mechanical sciences 47(2) (2005) 293-302.

[23] O.M. Querin, M. Victoria, P. Martí, Topology optimization of truss-like continua with different material properties in tension and compression, Structural and Multidisciplinary Optimization 42(1) (2010) 25-32.

[24] M. Victoria, O.M. Querin, P. Martí, Generation of strut-and-tie models by topology design using different material properties in tension and compression, Structural and Multidisciplinary Optimization 44(2) (2011) 247-258. 
[25] O. Amir, A topology optimization procedure for reinforced concrete structures, Computers \& Structures 114 (2013) 46-58.

[26] S. Liu, H. Qiao, Topology optimization of continuum structures with different tensile and compressive properties in bridge layout design, Structural and Multidisciplinary Optimization 43(3) (2011) 369-380.

[27] K. Cai, A simple approach to find optimal topology of a continuum with tension-only or compression-only material, Structural and Multidisciplinary Optimization 43(6) (2011) 827-835.

[28] K. Cai, J. Cao, J. Shi, L. Liu, Q.H. Qin, Optimal layout of multiple bi-modulus materials, Structural and Multidisciplinary Optimization (2015) 1-11.

[29] K. Cai, Z. Gao, J. Shi, Topology optimization of continuum structures with bi-modulus materials, Engineering Optimization 46(2) (2014) 244-260.

[30] K. Cai, Q.H. Qin, Z. Luo, A.J. Zhang, Robust topology optimisation of bi-modulus structures, Computer-Aided Design 45(10) (2013) 1159-1169.

[31] W. Prager, A problem of optimal design, Proceedings of the union of theoretical and applied mechanics, 1958.

[32] G. Hegemier, W. Prager, On michell trusses, International Journal of Mechanical Sciences 11(2) (1969) 209-215.

[33] N.M. Ghoniem, E.P. Busso, N. Kioussis, H. Huang, Multiscale modelling of nanomechanics and micromechanics: an overview, Philosophical magazine 83(31-34) (2003) 3475-3528.

[34] A. Misra, P. Poorsolhjouy, Granular micromechanics model for damage and plasticity of cementitious materials based upon thermomechanics, Mathematics and Mechanics of Solids (2015) 1081286515576821.

[35] A. Misra, P. Poorsolhjouy, Granular micromechanics based micromorphic model predicts frequency band gaps, Continuum Mechanics and Thermodynamics 28(1-2) (2016) 215-234.

[36] A. Misra, V. Singh, Thermomechanics-based nonlinear rate-dependent coupled damage-plasticity granular micromechanics model, Continuum Mechanics and Thermodynamics 27(4-5) (2015) 787-817. [37] M.P. Bendsøe, O. Sigmund, Topology optimization: theory, methods, and applications, Springer Science \& Business Media2013.

[38] A. Rietz, Sufficiency of a finite exponent in SIMP (power law) methods, Structural and Multidisciplinary Optimization 21(2) (2001) 159-163.

[39] Y. Xie, G.P. Steven, A simple evolutionary procedure for structural optimization, Computers \& structures 49(5) (1993) 885-896.

[40] X. Huang, Y.-M. Xie, A further review of ESO type methods for topology optimization, Structural and Multidisciplinary Optimization 41(5) (2010) 671-683.

[41] X. Huang, Y. Xie, Evolutionary topology optimization of continuum structures with an additional displacement constraint, Structural and Multidisciplinary Optimization 40(1-6) (2010) 409-416.

[42] X. Huang, M. Xie, Evolutionary topology optimization of continuum structures: methods and applications, John Wiley \& Sons2010.

[43] A. Misra, W. Ching, Theoretical nonlinear response of complex single crystal under multi-axial tensile loading, Scientific reports 3 (2013).

[44] A. Misra, L. Ouyang, J. Chen, W. Ching, Ab initio calculations of strain fields and failure patterns in silicon nitride intergranular glassy films, Philosophical Magazine 87(25) (2007) 3839-3852.

[45] A. Misra, S. Huang, Micromechanical stress-displacement model for rough interfaces: Effect of asperity contact orientation on closure and shear behavior, International Journal of Solids and Structures 49(1) (2012) 111-120.

[46] A. Misra, P. Poorsolhjouy, Micro-macro scale instability in 2D regular granular assemblies, Continuum Mechanics and Thermodynamics 27(1-2) (2015) 63-82. 
[47] A. Misra, Y. Yang, Micromechanical model for cohesive materials based upon pseudo-granular structure, International Journal of Solids and Structures 47(21) (2010) 2970-2981.

[48] A.-L. Cauchy, Sur l'equilibre et le mouvement d'un systeme de points materiels sollicites par des forces d'attraction ou de repulsion mutuelle, Excercises de Mathematiques 3 (1826-1830) 188-212.

[49] F. dell'Isola, U. Andreaus, L. Placidi, At the origins and in the vanguard of peri-dynamics, non-local and higher gradient continuum mechanics. An underestimated and still topical contribution of Gabrio Piola, Mechanics and Mathematics of Solids (2013).

[50] C.L. Navier, Sur les lois de l'equilibre et du mouvement des corps solides elastiques, Memoire de I'Academie Royale de Sciences 7 (1827) 375-393.

[51] A. Misra, P. Poorsolhjouy, Identification of higher-order elastic constants for grain assemblies based upon granular micromechanics, Mathematics and Mechanics of Complex Systems 3(3) (2015) 285-308. [52] A. Misra, P. Poorsolhjouy, Elastic Behavior of 2D Grain Packing Modeled as Micromorphic Media Based on Granular Micromechanics, Journal of Engineering Mechanics (2016) C4016005.

[53] F. Dell'Isola, T. Lekszycki, M. Pawlikowski, R. Grygoruk, L. Greco, Designing a light fabric metamaterial being highly macroscopically tough under directional extension: first experimental evidence, Zeitschrift für angewandte Mathematik und Physik 66(6) (2015) 3473-3498.

[54] L. Placidi, L. Greco, S. Bucci, E. Turco, N.L. Rizzi, A second gradient formulation for a 2D fabric sheet with inextensible fibres, Zeitschrift für angewandte Mathematik und Physik 67(5) (2016) 114.

[55] A. Della Corte, I. Giorgio, D. Scerrato, Pantographic 2D sheets: Discussion of some numerical investigations and potential applications, International Journal of Non-Linear Mechanics 80 (2016) 200208.

[56] F. dell'Isola, I. Giorgio, M. Pawlikowski, N. Rizzi, Large deformations of planar extensible beams and pantographic lattices: heuristic homogenization, experimental and numerical examples of equilibrium, Proc. R. Soc. A, The Royal Society, 2016, p. 20150790.

[57] A. Misra, V. Singh, Micromechanical model for viscoelastic materials undergoing damage, Continuum Mechanics and Thermodynamics 25(2-4) (2013) 343-358.

[58] A. Misra, V. Singh, Nonlinear granular micromechanics model for multi-axial rate-dependent behavior, International Journal of Solids and Structures 51(13) (2014) 2272-2282.

[59] A. Misra, P. Poorsolhjouy, Granular micromechanics model of anisotropic elasticity derived from Gibbs potential, Acta Mechanica 227(5) (2016) 1393-1413.

[60] S.I. Ranganathan, M. Ostoja-Starzewski, Universal elastic anisotropy index, Physical Review Letters 101(5) (2008) 055504.

[61] Q. Li, G.P. Steven, Y. Xie, O.M. Querin, Evolutionary topology optimization for temperature reduction of heat conducting fields, International Journal of Heat and Mass Transfer 47(23) (2004) 50715083.

[62] R. Ansola, E. Veguería, J. Canales, C. Alonso, Electro-thermal compliant mechanisms design by an evolutionary topology optimization method, Engineering Computations: Int J for Computer-Aided Engineering 30(7) (2013) 961-981.

[63] Y.M. Xie, X. Huang, Recent developments in evolutionary structural optimization (ESO) for continuum structures, IOP Conference Series: Materials Science and Engineering, IOP Publishing, 2010, p. 012196.

[64] K.-J. Bathe, Finite element procedures, Klaus-Jurgen Bathe2006.

[65] R.D. Cook, Concepts and applications of finite element analysis, John Wiley \& Sons2007.

[66] E. Bednarczyk, T. Lekszycki, A novel mathematical model for growth of capillaries and nutrient supply with application to prediction of osteophyte onset, Zeitschrift für angewandte Mathematik und Physik 67(4) (2016) 1-14.

[67] T. Lekszycki, Modelling of bone adaptation based on an optimal response hypothesis, Meccanica 37(4-5) (2002) 343-354. 
[68] T. Lekszycki, Functional adaptation of bone as an optimal control problem, Journal of Theoretical and Applied Mechanics 43(3) (2005) 555-574.

[69] Y. Lu, T. Lekszycki, A novel coupled system of non-local integro-differential equations modelling Young's modulus evolution, nutrients' supply and consumption during bone fracture healing, Zeitschrift für angewandte Mathematik und Physik 67(5) (2016) 111.

[70] J.L. Katz, A. Meunier, The elastic anisotropy of bone, Journal of Biomechanics 20(11-12) (1987) 1063-1070.

[71] M. Bruggi, A. Taliercio, Maximization of the fundamental eigenfrequency of micropolar solids through topology optimization, Structural and Multidisciplinary Optimization 46(4) (2012) 549-560. 


\section{Figure Captions}

Figure 1: Length scales, computational approach and demand.

Figure 2: Design domain for granular material structure topology optimization and its boundary conditions

Figure 3: Schematic of granular micromechanics approach.

Figure 4: The relationship of each pair of grains.

Figure 5: Geometry and boundary conditions of cantilever

Figure 6. Topology variation history (left column) and anisotropy evolution (right column) for $\lambda=0.4$ at selected iteration numbers $(8,12,22,32,47$ and 61$)$.

Figure 7: a. Displacement for the optimal structure. b. Von Mises stress fields, averaged stress distribution can be seen in final topology. c. First principle stress indicator for optimal topology, red region indicate that the material is in tension, blue in compression.

Figure 8: Iteration history of objective and constraint function

Figure 9: Topology variation, objective function and constraint for different $\lambda$. Red and blue indicate tensile and compressive major principal stress regions, respectively, on the final topology.

Figure 10: Objective function and final topology for different volume constraint

Figure 11: Geometry and boundary conditions of corner loading cantilever

Figure 12: Topology variation history with optimization iteration for $\lambda=0.3$

Figure 13: Topology variation, objective function and constraint for different $\lambda$.

Figure 14: Geometry and boundary conditions of Michell structure

Figure 15: Topology variation history with optimization iteration for $\lambda=0.2$

Figure 16: Topology variation, objective function and constraint for different $\lambda$. Red and blue indicate tensile and compressive major principal stress regions, respectively, on the final topology.

Figure 17: Geometry and boundary conditions of MBB structure

Figure 18: Topology variation history of MBB structure with optimization iteration for $\lambda=0.2$

Figure 19: Topology variation, objective function and constraint for different $\lambda$. Color shows uniform distribution of von Mises stress in the final topology. 


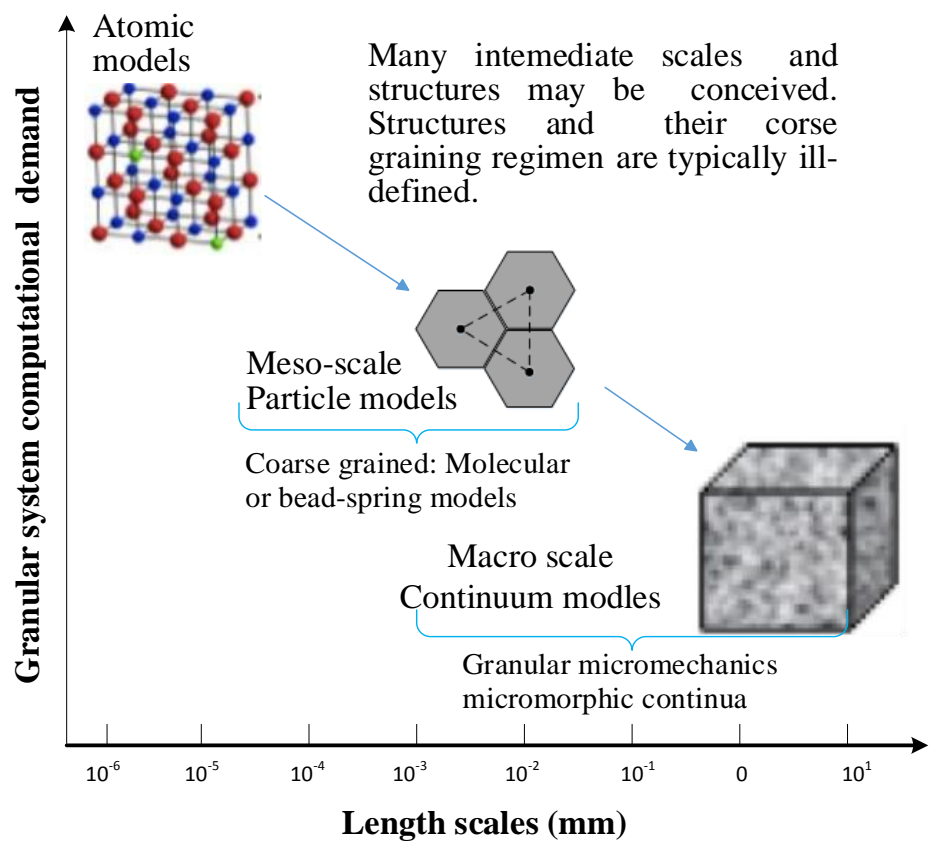

Figure 1: Length scales, computational approach and demand. 


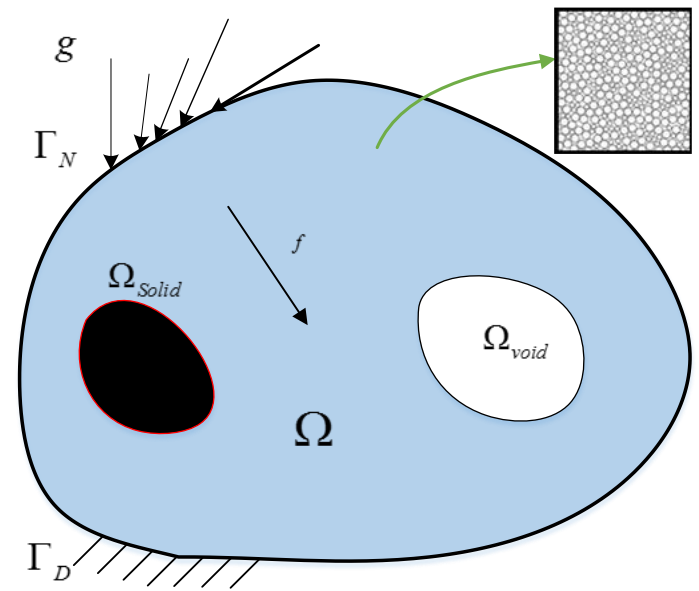

Figure 2: Design domain for granular material structure topology optimization and its boundary conditions 


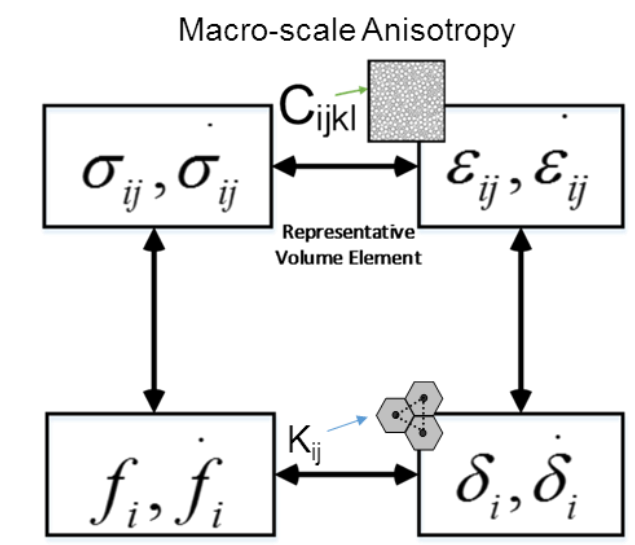

Micro-scale Tension-Compression Asymmetry

Figure 3: Schematic of granular micromechanics approach. 


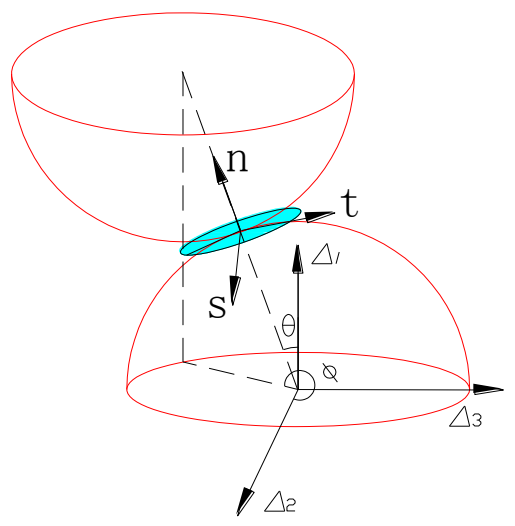

Figure 4: The relationship of each pair of grains. 


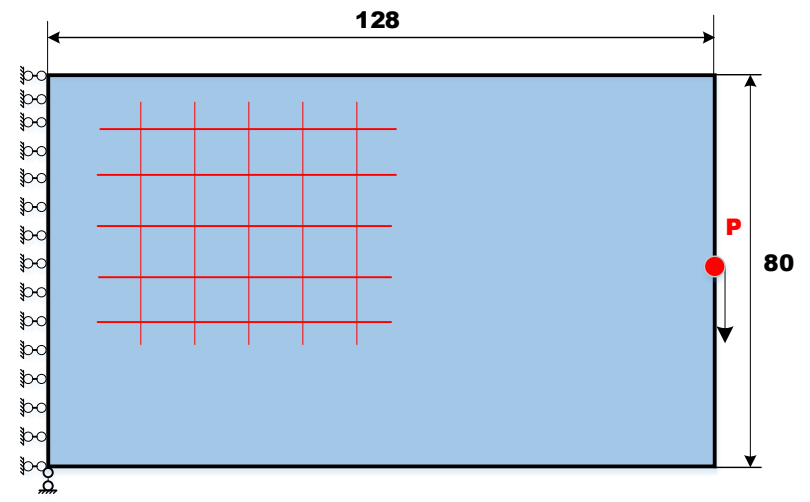

Figure 5: Geometry parameters and boundary conditions of cantilever plate 

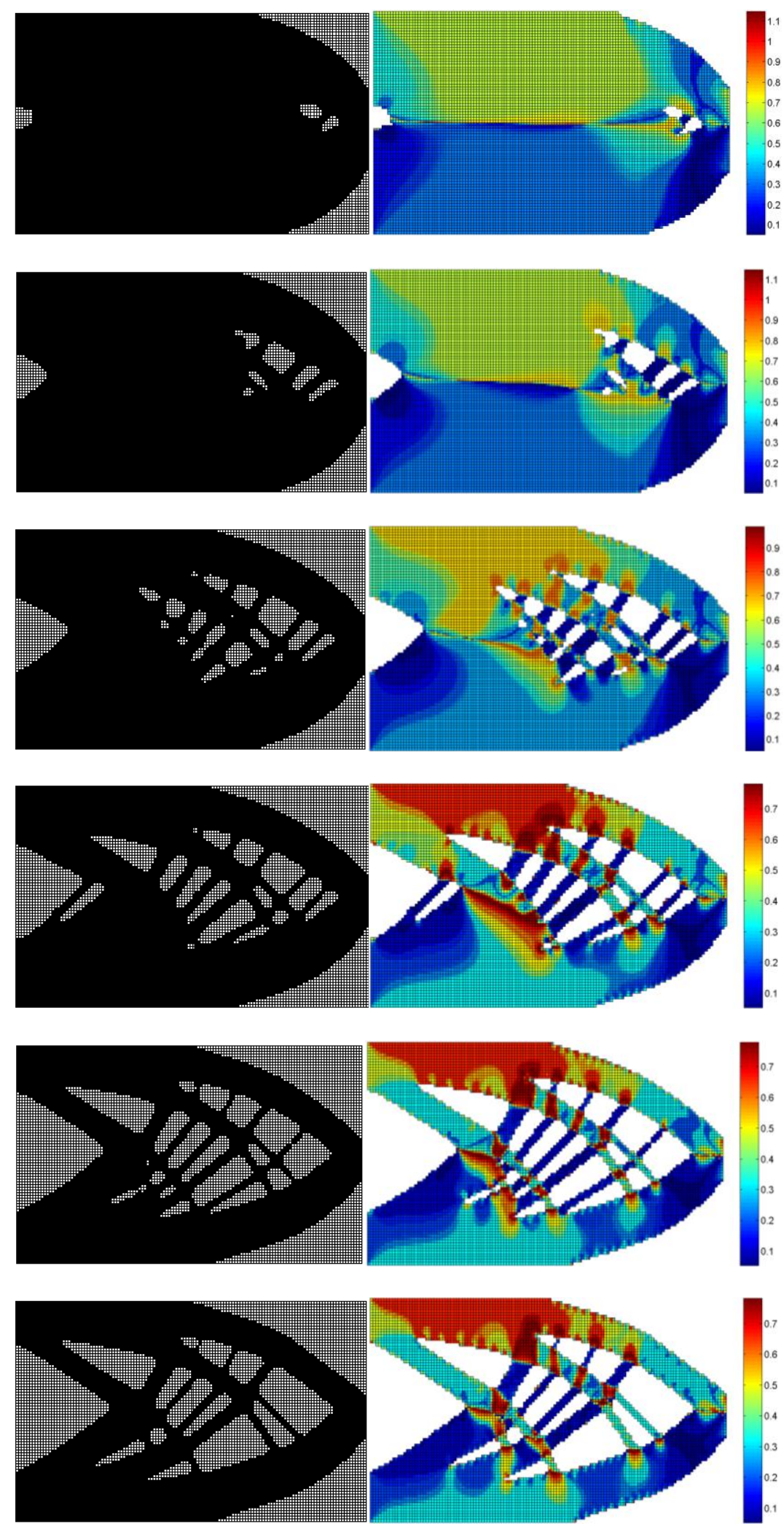

Figure 6: Topology variation history (left column) and anisotropy evolution (right column) for $\lambda=0.4$ at selecteiteration numbers $(8,12,22,32,47$ and 61$)$. 

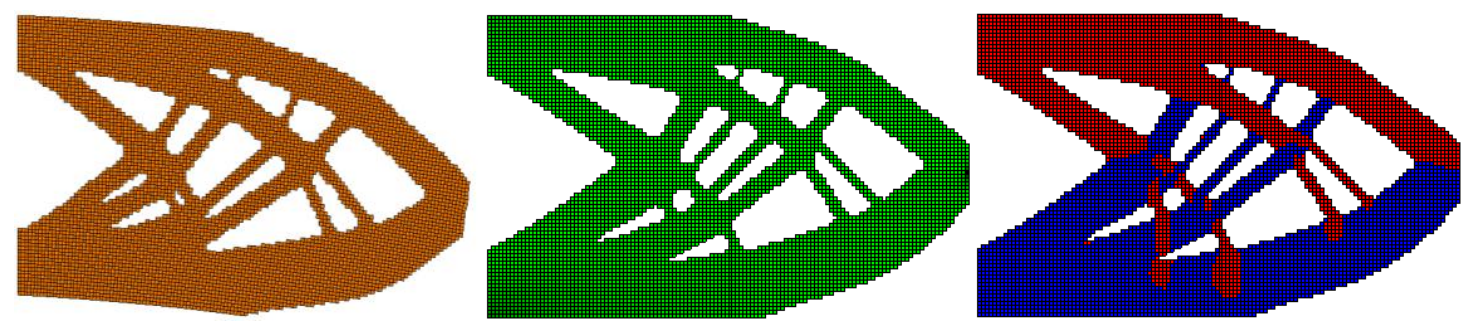

Figure 7: a. Displacement for the optimal structure. b. Von Mises stress fields, averaged stress distribution can be seen in final topology. c. First principle stress indicator for optimal topology, red region indicate that the material is in tension, blue in compression. 


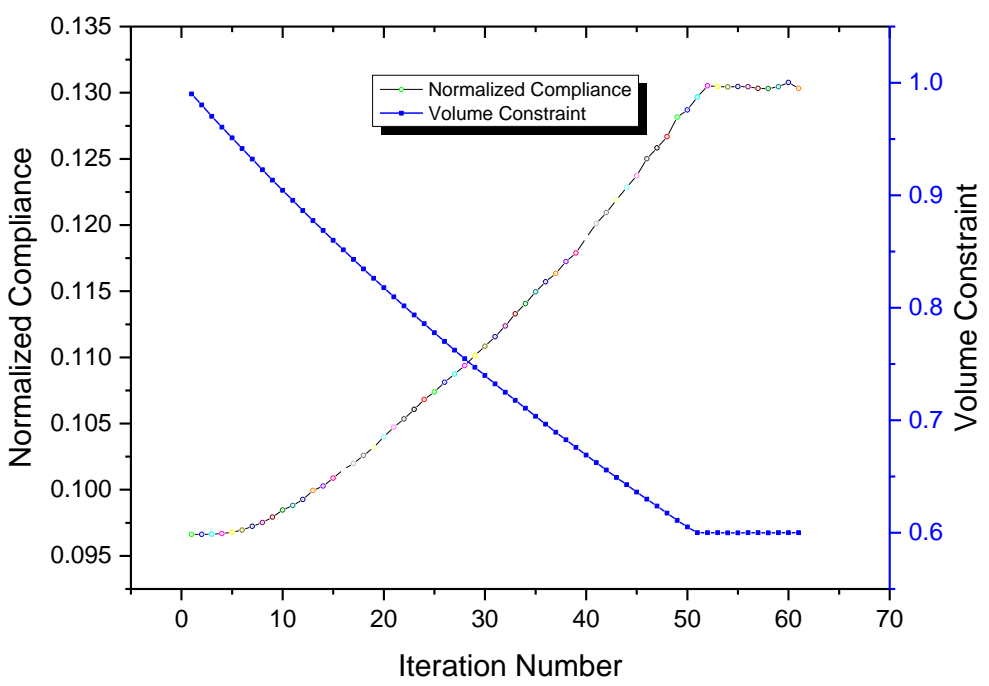

Figure 8: Iteration history of objective and constraint function 


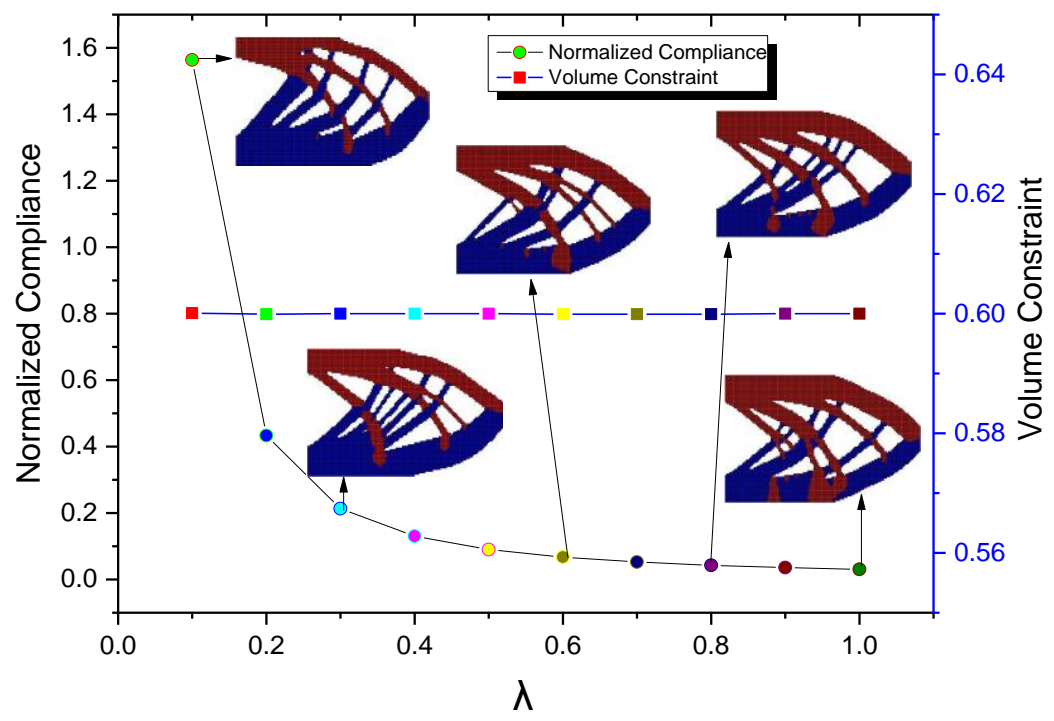

Figure 9: Topology variation, objective function and constraint for different $\lambda$. Red and blue indicate tensile and compressive major principal stress regions, respectively, on the final topology. 


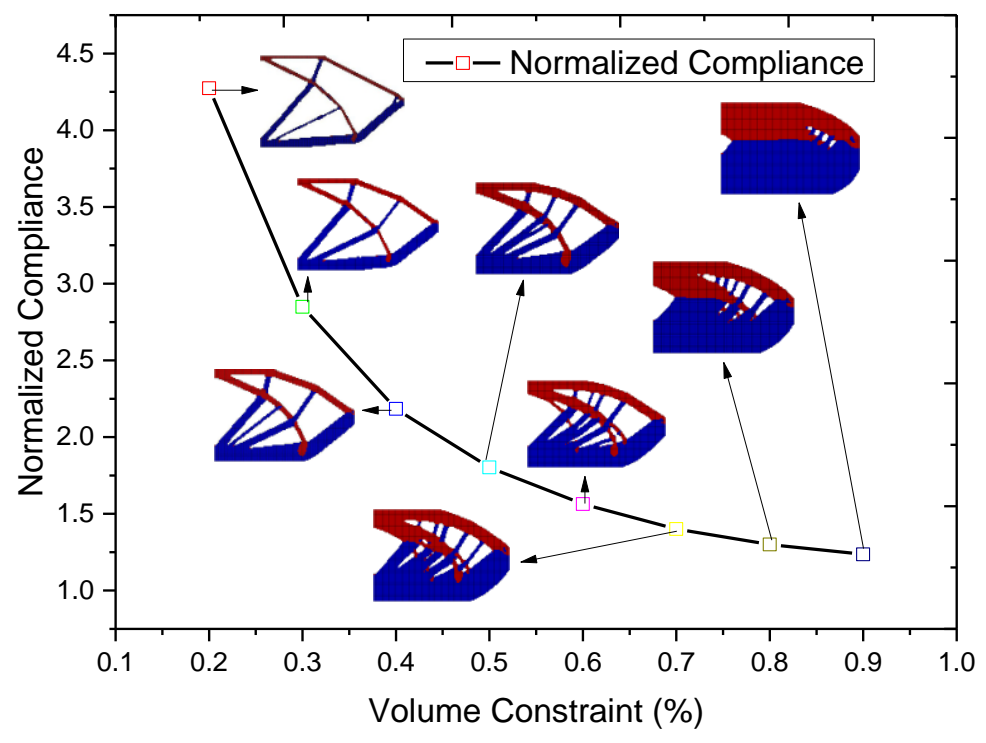

Figure 10: Objective function and final topology for different volume constraint. 


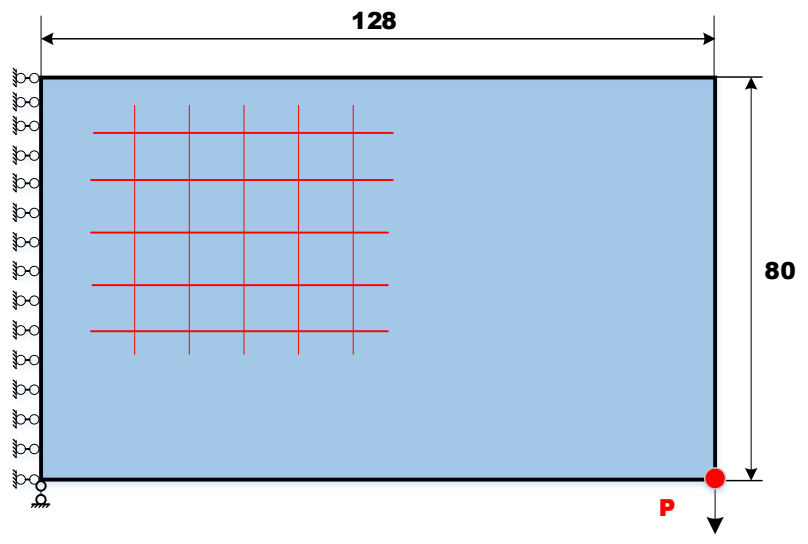

Figure 11: Geometry and boundary conditions of corner loading cantilever 


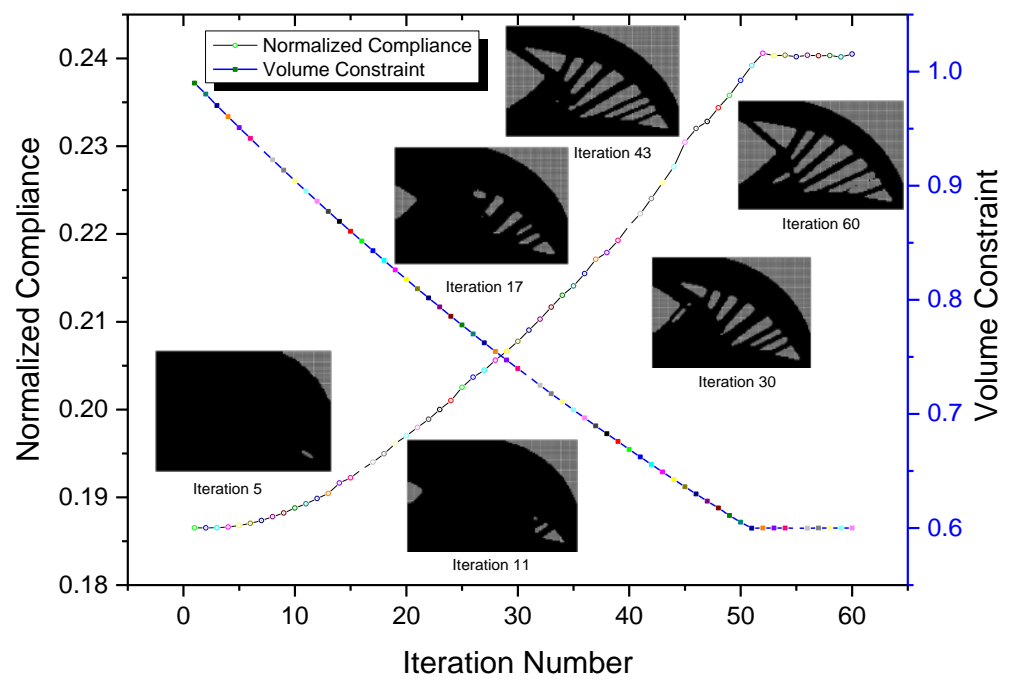

Figure 12: Topology variation history with optimization iteration for $\lambda=0.3$ 


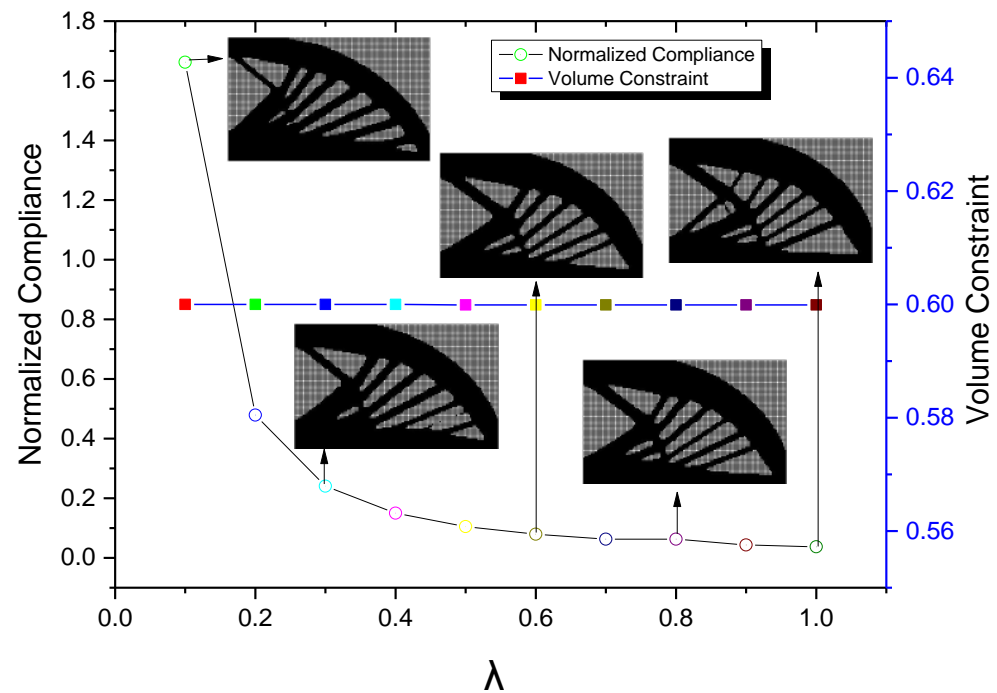

Figure 13: Topology variation, objective function and constraint for different $\lambda$. 


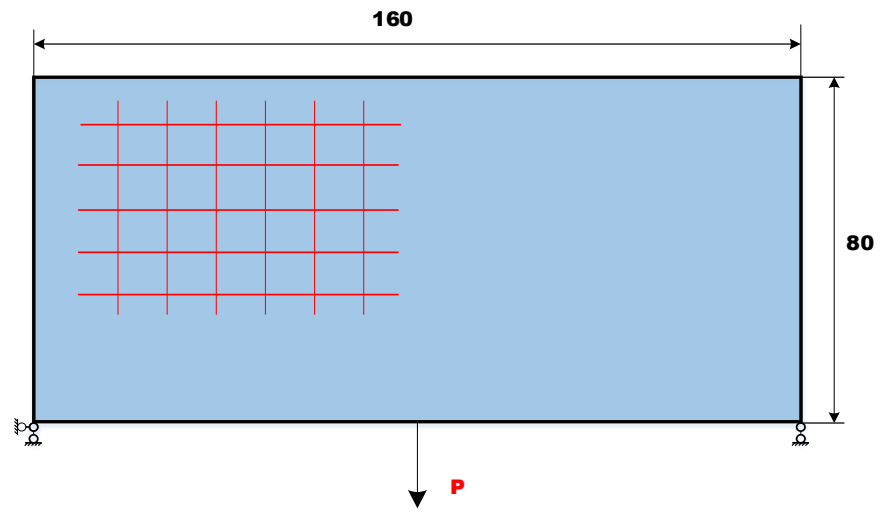

Figure 14: Geometry and boundary conditions of Michell structure 


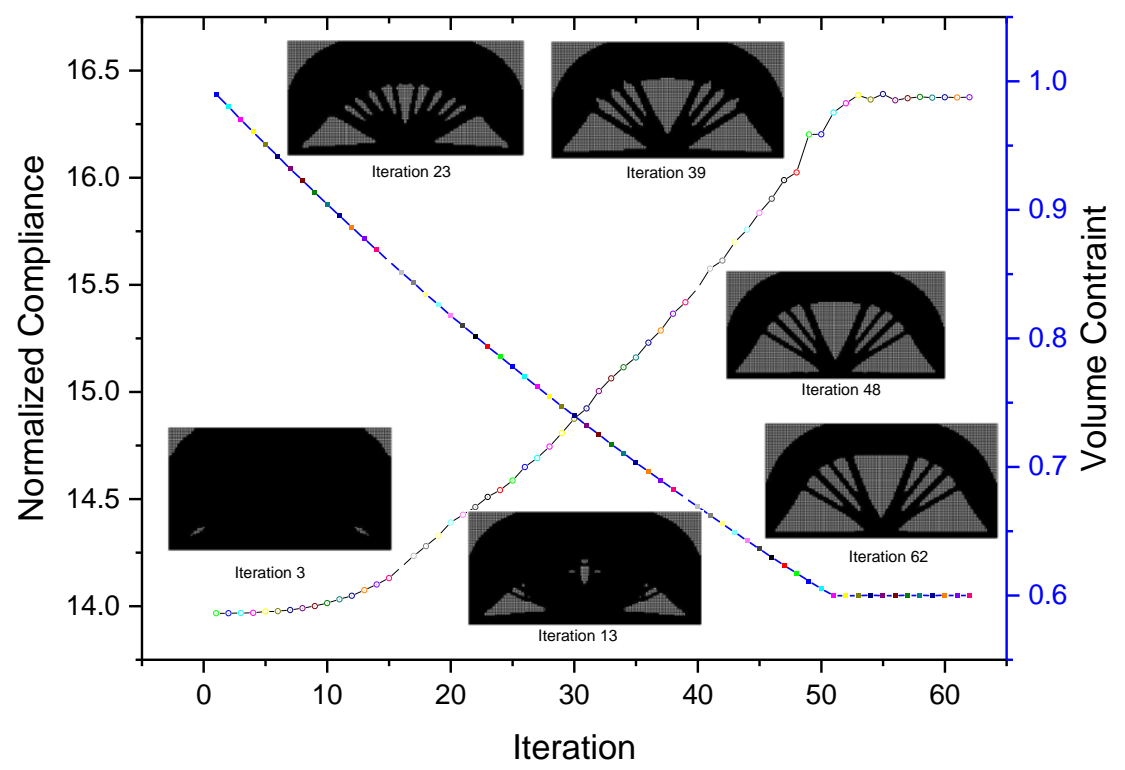

Figure 15: Topology variation history with optimization iteration for $\lambda=0.2$ 


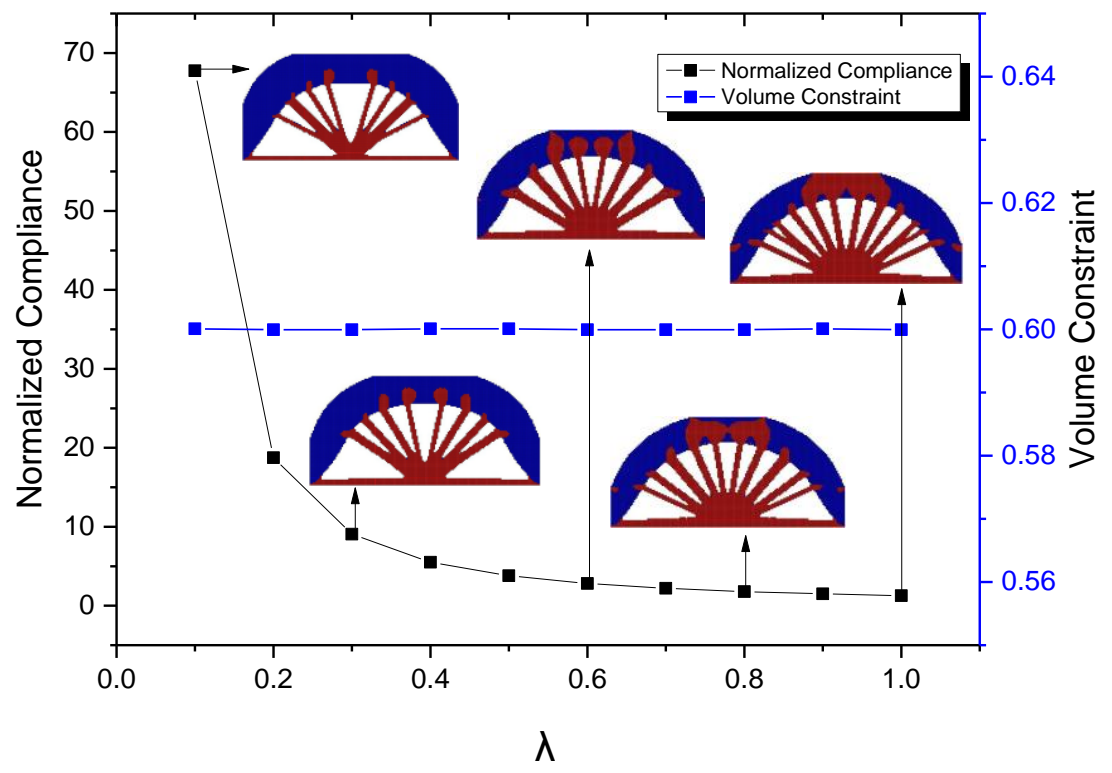

Figure 16: Topology variation, objective function and constraint for different $\lambda$. Red and blue indicate tensile and compressive major principal stress regions, respectively, on the final topology. 


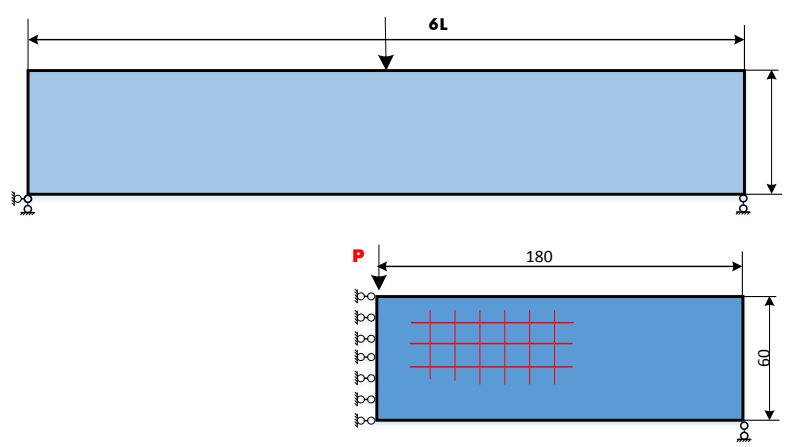

Figure 17: Geometry and boundary conditions of MBB structure 


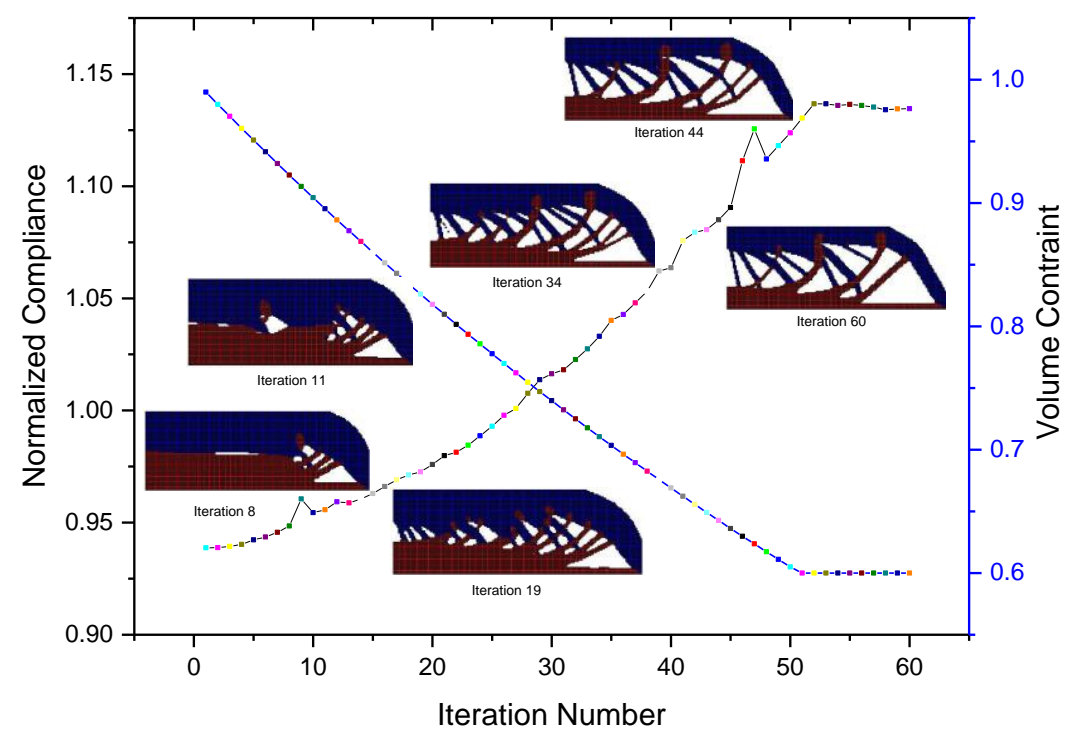

Figure 18: Topology variation history of MBB structure with optimization iteration for $\lambda=0.2$ 


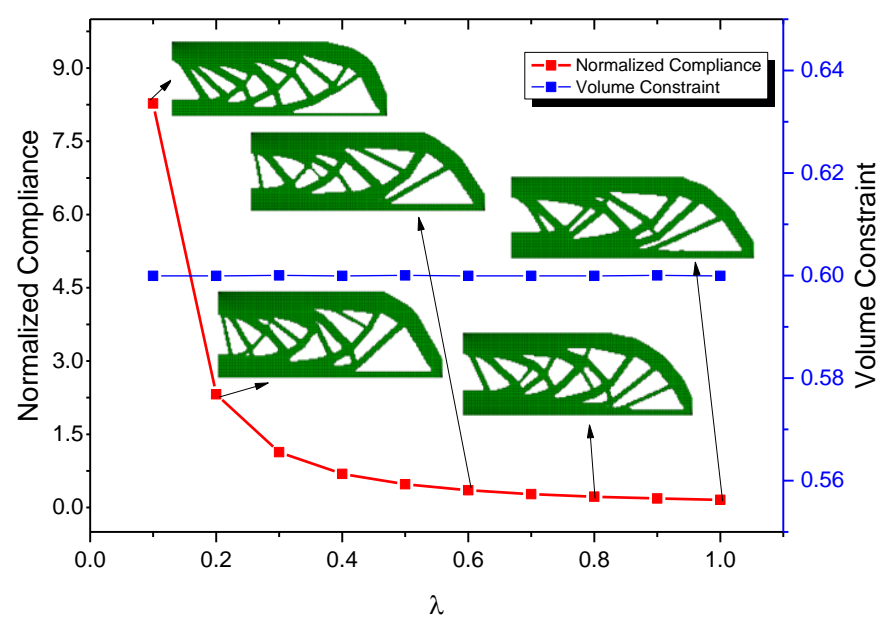

Figure 19: Topology variation, objective function and constraint for different $\lambda$. Color shows uniform distribution of von Mises stress in the final topology. 

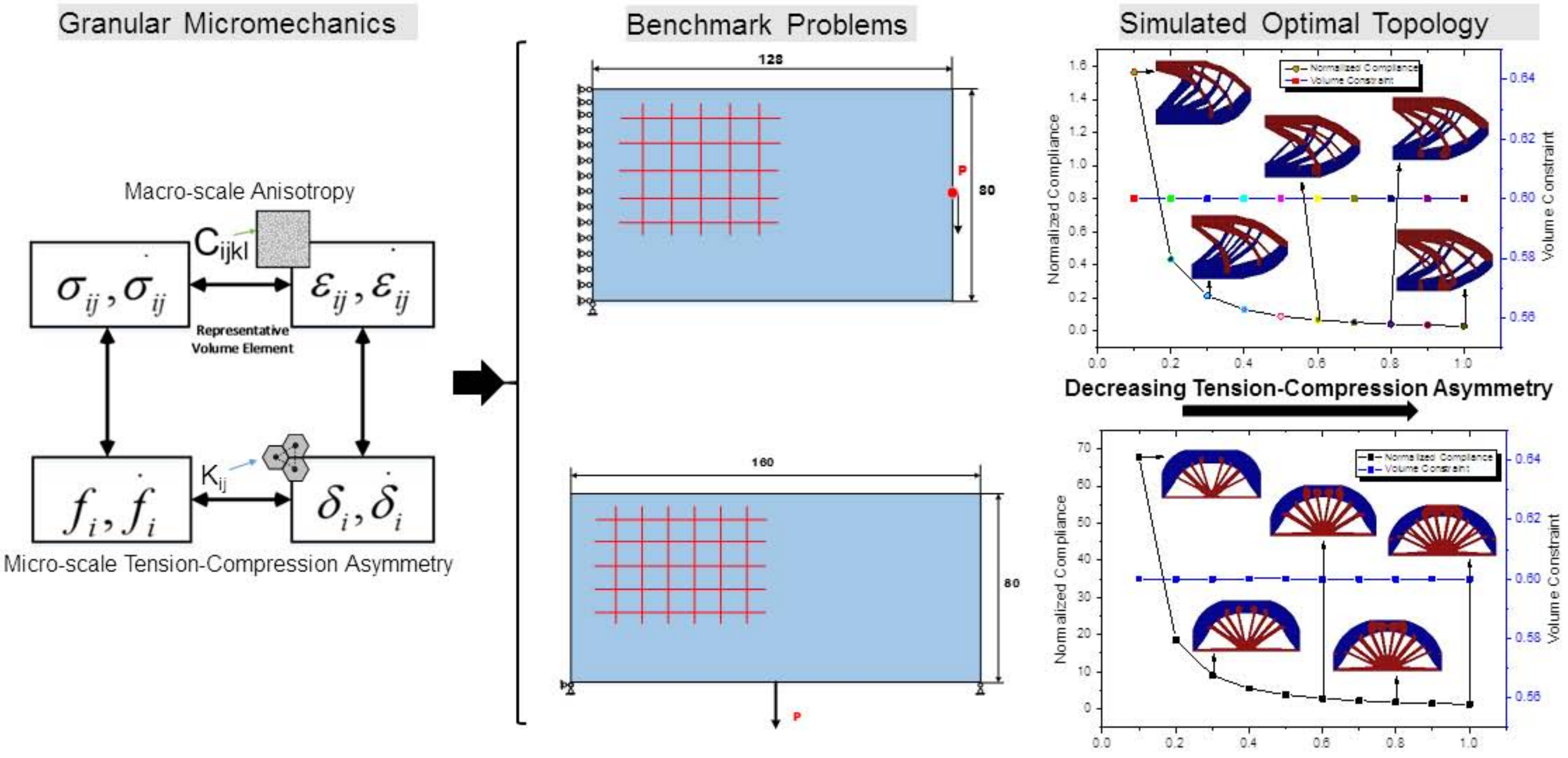